\title{
Unbiased Estimation of Reliability Function from a Mixture of Two Exponential Distributions Based on a Single Observation
}

\author{
Keyu Nie ${ }^{1}$ Bikas.K. Sinha ${ }^{2}$ and A.S. Hedayat ${ }^{3}$ \\ Department of Mathematics, Statistics, and Computer Science \\ University of Illinois at Chicago
}

\begin{abstract}
In this paper we investigate an aspect of unbiased estimation of the reliability function, based on a single observation from a mixture of two exponential distributions with known mixing proportions. We introduce a few equivalent versions of unbiased estimators, and find that they give proper estimates only if negative weights on component mixture distributions are allowed.
\end{abstract}

Keywords: Exponential Distribution, Mixture Distribution, Proper Mixture, Reliability Function, Unbiased Estimation, UMVUE, Negative Weights,

\section{Introduction}

In the study of life testing and reliability analysis, one important aspect is to find estimates of parametric functions for the underlying life distribution. For practical purposes, it is desirable to obtain an unbiased estimate of the reliability function (survival function) $R(t)=e^{-t / \lambda}$ for a specified $t>0$, supposing the real life distribution follows exponential distribution.

Let $X_{1}, \ldots, X_{n}$ be $n$ independent random variables following exponential distribution with mean $\lambda(>0)$ unknown, written henceforth as $\exp (\lambda)$. Pugh (1963) and Basu (1964) proposed the uniformly minimum variance unbiased

\footnotetext{
${ }^{1}$ keyunie@gmail.com

2 bikassinha1946@gmail.com
}

33hedayat@uic.edu 
estimator (UMVUE) of the reliability function based on the complete random sample of size n:

$$
\hat{R}_{\text {umvue }}(t)= \begin{cases}\left(1-\frac{t}{W}\right)^{n-1} & W>t \\ 0 & \text { otherwise }\end{cases}
$$

where $W=\sum_{i=1}^{n} X_{i}$.

For cases involving initial $r(\leq n)$ order statistics $\left(X_{(1)}, \ldots, X_{(r)}\right)$ instead of a complete random set of size $n$, Basu (1964) developed the complementary version of the UMVUE of $R(t)$ as:

$$
\hat{R}_{o s}(t)= \begin{cases}\left(1-\frac{t}{W^{*}}\right)^{r-1} & W^{*}>t \\ 0 & \text { otherwise }\end{cases}
$$

where $W^{*}=\sum_{i=1}^{r} X_{(i)}+(n-r) X_{(r)}$. Apparently, $\hat{R}_{u m v u e}(t)$ is a special form of $\hat{R}_{o s}(t)$ by letting $r=n$.

Sinha et al. (2006) derived unique and explicit form of the unbiased estimator of $R(t)$ based on a single $i^{t h}$ order statistic $X_{(i)}(1 \leq i \leq n)$. We cite a customized format with $i=2$ from their formula for completeness and our convenience.

$$
h_{2}\left(Z_{(2)}\right)=\sum_{y_{1}=0}^{\infty} \frac{1}{n \alpha^{y_{1}}} I\left(Z_{(2)}>\alpha^{y_{1}} t\right),
$$

where $Z_{(2)}=(n-1) X_{(2)}$ and $\alpha=\frac{n}{n-1}$. More details can be retrieved from their paper. Based on their result, one may obtain a formula for unbiased estimate of $R(t)$ based on $k$ selected order statistics $\left(X_{\left(i_{1}\right)}, \ldots, X_{\left(i_{k}\right)}, 1 \leq\right.$ $\left.i_{1}<\cdots<i_{k} \leq n\right)$ only.

In this paper, we identify and investigate the nature of unbiased estimator of $R(t)$ from a mixture of two exponential distributions based on a single observation. We assume that prior knowledge of the weights of the mixture distributions and the ratio of the means of the two distributions are available from previous studies. In section 2 we shall introduce a few equivalent versions of unbiased estimators, and establish that any unbiased estimator of $R(t)$ based on a random observation from a mixture of two exponential distributions remains a proper estimator (between 0 and 1 ) if and only if one of the two weights of the mixture distributions is negative. In section 3 , we proceed to construct unbiased estimates for the variance of these estimators but limit the discussion to proper estimators only. 
It must be noted that the concept of "mixture distribution" in this paper is used in the broadest sense. Mathematically, we intentionally include negative weights as long as the density function is properly defined. Some motivating examples are raised by Ross (2010) for instance the life distribution of parallel system of two independent components with $\exp (\lambda)$ is the maximum of two component lives, given by $2 \exp (\lambda)-\exp \left(\frac{\lambda}{2}\right)$. Jevremovic (1991) pointed out that, by using an auto regressive or moving average process, negative weight of mixture of two exponentials occurred naturally.

$$
Y_{t}= \begin{cases}\alpha \xi_{t} & w \cdot p \cdot p_{0} \\ \beta \xi_{t}+Y_{t-1} & w \cdot p \cdot p_{1} \\ Y_{t-1} & w \cdot p \cdot q_{1}\end{cases}
$$

where $\alpha, \beta, p_{0}, p_{1}, q_{1}$ are between 0 and 1 , and $p_{0}+p_{1}+q_{1}=1$. The sequence $\xi_{t}$ is an i.i.d sequence following $\exp (\lambda)$, then $Y_{t}$ follows a mixture exponential

distributions: $f_{Y}(y)=a_{1} \exp \left(\gamma_{1}\right)+a_{2} \exp \left(\theta \gamma_{1}\right)$, with $\gamma_{1}=\alpha \lambda, \theta=\frac{\beta\left(1-q_{1}\right)}{p_{0} \alpha}$, $a_{1}=p_{0}(\beta-\alpha) /\left(\left(1-q_{1}\right) \beta-p_{0} \alpha\right)$, and $a_{2}=1-a_{1}$.

\section{Improper Unbiased Estimates Based On A Single Observation}

We suppose a random variable $X$ follows a mixture of two exponential distributions with corresponding means $\lambda$ and $\theta \lambda(\theta, \lambda>0)$; denote it as $X \sim p \exp (\lambda)+q \exp (\theta \lambda)$, where $p+q=1, p$ and $\theta$ are known and $\lambda$ is unknown. The problem is to unbiasedly estimate the function $R(t)=e^{-t / \lambda}$ for a given $t>0$, based on a single observation $x$.

It is clear that indicator function is unbiased estimator of $R(t)$ for exponential random variable. However a comparable unbiased estimator of $R(t)$ for a mixture of two exponential distributions has not been investigated in the literature. Inspired by Sinha et al. (2006), the following theorem provides a formula of such unbiased estimator.

Theorem 2.1. Any unbiased estimator of $R(t)$ denoted $h(x ; t)$ should satisfy $I(x>t)=p h(x ; t)+q h(\theta x ; t)$, where $I(x>t)$ is an indicator function.

Proof. 


$$
\begin{aligned}
& \frac{1}{\lambda} \int_{0}^{\infty} I(x>t) e^{-\frac{x}{\lambda}} d x=R(t)=E(h(x ; t)) \\
& =\frac{1}{\lambda} \int_{0}^{\infty} h(x ; t)\left(p e^{-\frac{x}{\lambda}}+\frac{q}{\theta} e^{-\frac{x}{\theta \lambda}}\right) d x \\
& =\frac{1}{\lambda}\left(\int_{0}^{\infty} p h(x ; t) e^{-\frac{x}{\lambda}}+\int_{0}^{\infty} q h(\theta x ; t) e^{-\frac{x}{\lambda}}\right) d x
\end{aligned}
$$

So $I(x>t)=p h(x ; t)+q h(\theta x ; t)$ follows from the completeness property of the exponential distribution.

Theorem 2.1 shows implicit relation between $h(x ; t)$ and $I(x>t)$ in a nested way. The function $h(x ; t)$ can be explicitly formalized by iteration within the nested function. There are several variants of $h(x ; t)$. We propose a couple of basic versions $\left(h_{1}(x ; t)\right.$ and $\left.h_{2}(x ; t)\right)$ here.

$$
h_{1}(x ; t)=\sum_{k=0}^{\infty} \frac{1}{p}\left(-\frac{q}{p}\right)^{k} I\left(X>\theta^{-k} t\right),
$$

and

$$
h_{2}(x ; t)=\sum_{k=0}^{\infty} \frac{1}{q}\left(-\frac{p}{q}\right)^{k} I\left(X>\theta^{k+1} t\right) .
$$

The steps in Theorem 2.2 illustrate the process of how $h_{1}(x ; t)$ and $h_{2}(x ; t)$ are constituted. Obviously, $h_{1}(x ; t)$ and $h_{2}(x ; t)$ are mutually convertible by swapping $p$ and $q$ as well as replacing $\theta$ with $\frac{1}{\theta}$. Theorem 2.2 also explains $h_{1}(x ; t)$ and $h_{2}(x ; t)$ are unbiased estimators. Notice that any linear combination of these two estimators is also an unbiased estimator of $R(t)$.

Theorem 2.2. $E\left(h_{1}(x ; t)\right)=E\left(h_{2}(x ; t)\right)=R(t)$.

Proof.

Based on the result of Theorem 2.1, it is trivial to show that:

$$
E(I(X>t))=p R(t)+q R\left(\theta^{-1} t\right)
$$

Bringing $R(t)$ to the left side of the equation and substituting within the 
Table 1: Convergence of $h_{1}(x ; t)$ and $h_{2}(x ; t)$

\begin{tabular}{|c|c|c|}
\hline & $\theta<1$ & $\theta>1$ \\
\hline$\left|\frac{q}{p}\right|<1$ & $h_{1}(x ; t)$ & $h_{1}(x ; t), h_{2}(x ; t)$ \\
\hline$\left|\frac{q}{p}\right|>1$ & $h_{1}(x ; t), h_{2}(x ; t)$ & $h_{2}(x ; t)$ \\
\hline$\left|\frac{q}{p}\right|=1$ & $h_{1}(x ; t)$ & $h_{2}(x ; t)$ \\
\hline
\end{tabular}

nested function on the right side repeatedly, we derive the following equation.

$$
\begin{aligned}
R(t) & =E\left(\frac{1}{p} I(X>t)\right)-\frac{q}{p} R\left(\theta^{-1} t\right)=\ldots \ldots \\
& =E\left(\sum_{k=0}^{\infty} \frac{1}{p}\left(-\frac{q}{p}\right)^{k} I\left(X>\theta^{-k} t\right)\right)=E\left(h_{1}(x ; t)\right) .
\end{aligned}
$$

Again,

$$
E(I(X>\theta t))=p R(\theta t)+q R(t)
$$

This yields:

$$
\begin{aligned}
R(t) & =E\left(\frac{1}{q} I(X>\theta t)\right)-\frac{p}{q} R(\theta t)=\ldots \ldots \\
& =E\left(\sum_{k=0}^{\infty} \frac{1}{q}\left(-\frac{p}{q}\right)^{k} I\left(X>\theta^{k+1} t\right)\right)=E\left(h_{2}(x ; t)\right) .
\end{aligned}
$$

When $x$ is recorded, one of $h_{1}(x ; t)$ and $h_{2}(x ; t)$ is an infinite sum of geometric series in view of $\theta^{-1}<1$ or $\theta<1$ respectively. If the absolute value of common ratio $\left(-\frac{q}{p}\right.$ or $\left.-\frac{p}{q}\right)$ goes beyond 1 , this infinite sum approaches infinity. It is very important ensuring both $h_{1}(x ; t)$ and $h_{2}(x ; t)$ being finite range mappings, especially between 0 and 1 . Table 1 presents their convergence based on $\left|\frac{q}{p}\right|$ and $\theta$.

According to Table 1 , the accessibility of $h_{1}(x ; t)$ or $h_{2}(x ; t)$ varies depending on $\left|\frac{q}{p}\right|$ and $\theta$. In the following, we argue that whether or not $h_{1}(x ; t) / h_{2}(x ; t)$ is proper $([0,1])$ unbiased estimator exists when they are accessible. In summary, three scenarios partitioned by $p(p>1, p<0$, $0<p<1$ ) are carefully examined. 
Table 2: $\theta=1 / 3, t=1, p=1.5, q=-0.5$

\begin{tabular}{|c|c|c|}
\hline Range of $x$ & $h_{1}(x ; t)$ & $h_{2}(x ; t)$ \\
\hline$\ldots$ & $\ldots$ & $\ldots$ \\
\hline$(3)^{-3}<x<(3)^{-2}$ & 0 & NA \\
\hline$(3)^{-2}<x<(3)^{-1}$ & 0 & NA \\
\hline$(3)^{-1}<x<1$ & 0 & NA \\
\hline $1<x<3$ & $\frac{2}{3}$ & NA \\
\hline $3<x<3^{2}$ & $\frac{2}{3}+\frac{2}{3} * \frac{1}{3}=\frac{8}{9}$ & NA \\
\hline $3^{2}<x<3^{3}$ & $\frac{2}{3}+\frac{2}{3} * \frac{1}{3}+\frac{2}{3} *\left(\frac{1}{3}\right)^{2}=\frac{26}{27}$ & NA \\
\hline$\ldots$ & $\ldots$ & $\ldots$ \\
\hline
\end{tabular}

1. $\mathbf{p}>$ 1. It follows with $-p<q=(1-p)<0$. The density function $\frac{p}{\lambda} e^{-\frac{x}{\lambda}}+\frac{q}{\theta \lambda} e^{-\frac{x}{\theta \lambda}}$ being non-negative, it requires $\theta<1$ too. We illustrate it by contradiction. As if $\theta>1$, the range of density function spans to negative infinity:

$$
\frac{p}{\lambda} e^{-\frac{x}{\lambda}}+\frac{q}{\theta \lambda} e^{-\frac{x}{\theta \lambda}}=e^{-\frac{x}{\lambda}}\left(\frac{p}{\lambda}+\frac{q}{\theta \lambda} e^{\frac{x}{\lambda}\left(1-\frac{1}{\theta}\right)}\right) \stackrel{x \rightarrow \infty}{\longrightarrow}-\infty
$$

Table 1 suggests that $h_{1}(x ; t)$ is the exclusive unbiased estimator supporting $\left|\frac{q}{p}\right|<1$ and $\theta<1$. We further claim $h_{1}(x ; t)$ is properly ranged $([0,1])$, in the light of common ratio $-\frac{q}{p}$ and first term $\frac{1}{p}$ yielding between 0 and 1 . Overall $h_{1}(x ; t)$ is a proper unbiased estimator for $R(t)$. The readers are encouraged to go through the example in Table $2(\theta=1 / 3, t=1, p=1.5, q=-0.5)$ to gain insight into more details.

2. $\mathbf{p}<\mathbf{0}$. Following similar approach as above, we obtain $\left|\frac{q}{p}\right|>1$ and $\theta>1$. We confirm that $h_{2}(x ; t)$ is the only proper unbiased estimator for $R(t)$ in this case.

3. $\mathbf{1}>\mathbf{p}>\mathbf{0}$. Therefore $1>q>0$ and the common ratio $-\frac{q}{p}$ of $h_{1}(x ; t)$ and $-\frac{p}{q}$ of $h_{2}(x ; t)$ are negative values. We prove that proper unbiased estimator does not exist. It is effortless to verify that $h_{1}(x ; t)$ or $h_{2}(x ; t)$ is not proper unbiased estimator using these facts: first term is greater than 1, infinite summation equals 1 and common ratio is negative. Further we establish that any linear combination $\phi h_{1}(x ; t)+(1-\phi) h_{2}(x ; t)$ is not a proper unbiased estimator either. Theorem 2.3 provides a detailed portion of the proof. A supporting example with $\theta=1.5^{-1}$, $t=1, p=\frac{1}{3}, q=\frac{2}{3}$ is also attached in Table 3 . 
Table 3: $\theta=1.5^{-1}, t=1, p=\frac{1}{3}, q=\frac{2}{3}$

\begin{tabular}{|c|c|c|}
\hline Range of $x$ & $h_{1}(x ; t)$ & $h_{2}(x ; t)$ \\
\hline$\ldots$ & $\ldots$ & $\ldots$ \\
\hline$(1.5)^{-3}<x<(1.5)^{-2}$ & 0 & $\left(\frac{p}{q}\right)^{2}=0.25$ \\
\hline$(1.5)^{-2}<x<(1.5)^{-1}$ & 0 & $-\frac{p}{q}=-0.5$ \\
\hline$(1.5)^{-1}<x<1$ & 0 & 1 \\
\hline $1<x<1.5$ & $\frac{1}{p}=3$ & 1 \\
\hline $1.5<x<1.5^{2}$ & $\frac{1}{p}-\frac{1}{p} * \frac{q}{p}=3-3 * 2=-3$ & 1 \\
\hline $1.5^{2}<x<1.5^{3}$ & $3-3 * 2+3 * 2^{2}=9$ & 1 \\
\hline$\ldots$ & $\ldots$ & $\ldots$ \\
\hline
\end{tabular}

Theorem 2.3. When $p, q>0$, there does not exist $\phi$ such that $\phi h_{1}(x ; t)+$ $(1-\phi) h_{2}(x ; t)$ is a proper unbiased estimator $([0,1])$ for $R(t)$.

Proof.

It is easy to check that $h_{1}(x ; t)$ and $h_{2}(x ; t)$ are improper estimators for the reason given before. Next we explain that the same conclusion continues to hold for any linear combination $\phi h_{1}(x ; t)+(1-\phi) h_{2}(x ; t)$ via contradiction.

Referring to Table 1 , there are only two particular cases where $h_{1}(x ; t)$ and $h_{2}(x ; t)$ become available at the same time:

$1\left|\frac{q}{p}\right|=\frac{q}{p}>1$ and $\theta>1$.

$20<\left|\frac{q}{p}\right|=\frac{q}{p}<1$ and $\theta<1$.

For the first case $\frac{q}{p}>1$ and $\theta>1$, we begin our arguments as follows. When an observed $x$ is greater than the specified $t>0, h_{1}(x ; t)$ is identical as constant 1 .

$$
h_{1}(x ; t)=\frac{\frac{1}{p}}{1+\frac{q}{p}}=1 .
$$

$h_{2}(x ; t)$ is finite sum up to the order of $k \geq 0$ if $x$ lies between $\theta^{k+1} t$ and $\theta^{k+2} t$.

$$
h_{2}(x ; t)=\frac{\frac{1}{q}}{1+\frac{q}{p}}\left(1-\left(-\frac{q}{p}\right)^{k}\right)=1-\left(-\frac{q}{p}\right)^{k},
$$

when $\theta^{k+1} t<x<\theta^{k+2} t$ and $k=0, \ldots, \infty$. 
Applying Equation 2.9 and 2.10, a linear combination of $h_{1}(x ; t)$ and $h_{2}(x ; t)$ can be written as follows. For $\theta^{k+1} t<x<\theta^{k+2} t, k=0, \ldots, \infty$

$$
\begin{aligned}
\phi h_{1}(x ; t)+(1-\phi) h_{2}(x ; t) & =\phi+(1-\phi)\left(1-\left(-\frac{q}{p}\right)^{k}\right) \\
& =1-(1-\phi)\left(-\frac{q}{p}\right)^{k}
\end{aligned}
$$

Equation 2.11 lies in the range $[0,1]$ if and only if $\phi=1$ in consideration of $\frac{q}{p}>1$. It suggests $h_{1}(x ; t)+(1-1) h_{2}(x ; t)=h_{1}(x ; t)$ would be the only solution, but our earlier claim verifies that $h_{1}(x ; t)$ is not a proper estimator. In this way, there does not exit proper estimator of $R(t)$ when $\frac{q}{p}>1$ and $\theta>1$.

We implement similar discussion in the other case $0<\frac{q}{p}<1$ and $\theta<1$ and draw the conclusion that there is no such $\phi$ that $\phi h_{1}(x ; t)+(1-\phi) h_{2}(x ; t)$ is a proper estimator of $R(t)$ when $p, q>0$.

In summary, it turns out that the proposed unbiased estimator of $R(t)$ becomes a proper estimator for $[p>1, q<0]$ or $[p<0, q>1]$, which implies a negative weight on mixture exponential distributions. For cases of positive weights only on mixture exponential distributions, additional information is needed in the case of providing a proper estimator of $R(t)$. One way of doing so is to observe each component of the mixture exponential distribution directly.

\section{Unbiased Estimators of the Variances of $h_{1}(x ; t)$ and $h_{2}(x ; t)$}

In this section, we shall suggest unbiased estimators of the variances of $h_{1}(x ; t)$ and $h_{2}(x ; t)$. Based on Section 2 , we focus our discussion on proper unbiased estimators only, which indicates two situations:

1. $\theta<1$, and $p>1$;

2. $\theta>1$, and $q>1(p<0)$.

We also investigate the properties of the variance estimator and successfully prove its non-negativeness for a special case.

Theorem 3.1. When $\theta<1$ and $p>1$, an unbiased estimator of the variance of $h_{1}(x ; t)$ is

$\hat{V}\left(h_{1}(x ; t)\right)=h_{1}^{2}(x ; t)-h_{1}(x / 2 ; t)$ 


$$
=\frac{1}{p^{2}} \sum_{k=1}^{\infty}\left(2 p\left(-\frac{q}{p}\right)^{k}+(1-2 p)\left(\frac{q}{p}\right)^{2 k}\right) I\left(X>\theta^{-k} t\right)-\sum_{k=0}^{\infty} \frac{1}{p}\left(-\frac{q}{p}\right)^{k} I\left(X>2 \theta^{-k} t\right) .
$$

Proof.

It is straightforward to show its unbiasedness as:

$$
\begin{aligned}
\operatorname{Var}\left(h_{1}(x)\right) & =E\left(h_{1}^{2}(x)\right)-E\left(h_{1}(x)\right)^{2} \\
& =E\left(h_{1}^{2}(x)\right)-R(t)^{2} \\
& =E\left(h_{1}^{2}(x)\right)-R(2 t) \\
& =E\left(h_{1}^{2}(x)\right)-E\left(h_{1}(x / 2)\right) \\
& =E\left(\hat{V}\left(h_{1}(x ; t)\right)\right) .
\end{aligned}
$$

We also note that:

$$
\begin{aligned}
& h_{1}^{2}(x ; t)=\frac{1}{p^{2}}\left\{\sum_{k=0}^{\infty}\left(\frac{q}{p}\right)^{2 k} I\left(X>\theta^{-k} t\right)+2 \sum \sum_{k>l=0}^{\infty}\left(-\frac{q}{p}\right)^{l+k} I\left(X>\theta^{-k} t\right)\right\} \\
& =\frac{1}{p^{2}}\left\{\sum_{k=0}^{\infty}\left(\frac{q}{p}\right)^{2 k} I\left(X>\theta^{-k} t\right)+2 \sum_{k=1}^{\infty}\left(-\frac{q}{p}\right)^{k} \frac{1-\left(-\frac{q}{p}\right)^{k}}{1+\frac{q}{p}} I\left(X>\theta^{-k} t\right)\right\} \\
& =\frac{1}{p^{2}}\left\{\sum_{k=0}^{\infty}\left(\frac{q}{p}\right)^{2 k} I\left(X>\theta^{-k} t\right)+2 p \sum_{k=0}^{\infty}\left(\left(-\frac{q}{p}\right)^{k}-\left(\frac{q}{p}\right)^{2 k}\right) I\left(X>\theta^{-k} t\right)\right\} \\
& =\frac{1}{p^{2}}\left\{\sum_{k=0}^{\infty}\left(2 p\left(-\frac{q}{p}\right)^{k}+(1-2 p)\left(\frac{q}{p}\right)^{2 k}\right) I\left(X>\theta^{-k} t\right)\right\} .
\end{aligned}
$$

So

$$
\begin{aligned}
& \hat{V}\left(h_{1}(x ; t)\right)=h_{1}^{2}(x ; t)-h_{1}(x / 2 ; t) \\
= & \frac{1}{p^{2}} \sum_{k=0}^{\infty}\left(2 p\left(-\frac{q}{p}\right)^{k}+(1-2 p)\left(\frac{q}{p}\right)^{2 k}\right) I\left(X>\theta^{-k} t\right)-\sum_{k=0}^{\infty} \frac{1}{p}\left(-\frac{q}{p}\right)^{k} I\left(X>2 \theta^{-k} t\right) .
\end{aligned}
$$

Theorem 3.2. When $\theta>1$ and $q>1$, an unbiased estimator of the variance of $h_{2}(x ; t)$ is $\hat{V}\left(h_{2}(x ; t)\right)=h_{2}^{2}(x ; t)-h_{2}(x / 2 ; t)$ 


$$
=\frac{1}{q^{2}} \sum_{k=0}^{\infty}\left(2 q\left(-\frac{p}{q}\right)^{k}+(1-2 q)\left(\frac{p}{q}\right)^{2 k}\right) I\left(X>\theta^{k+1} t\right)-\sum_{k=0}^{\infty} \frac{1}{q}\left(-\frac{p}{q}\right)^{k} I\left(X>2 \theta^{k+1} t\right) .
$$

Proof.

This proof can be achieved by switching the role of $p$ and $q$ as well as taking over $\theta$ with $\frac{1}{\theta}$ from the previous proof.

We are aware that $\hat{V}\left(h_{1}(x ; t)\right)$ and $\hat{V}\left(h_{2}(x ; t)\right)$ are feasible to negative region. On the grounds of complexity in formulas of $\hat{V}\left(h_{1}(x ; t)\right)$ and $\hat{V}\left(h_{2}(x ; t)\right)$, we only reason about its non-negative range for some special cases like $\theta=0.5$ (or 2). We check the sign of the proposed unbiased variance estimators through the following two Corollaries.

Corollary 3.3. When $\theta=0.5$ and $p>1$, an unbiased estimator of the variance of $h_{1}(x ; t)$ is

$\hat{V}\left(h_{1}(x ; t)\right)=\frac{1}{p^{2}} I(X>t)+\frac{1}{p^{2}} \sum_{k=1}^{\infty}\left(-(1+q)\left(-\frac{q}{p}\right)^{k-1}+(1-2 p)\left(\frac{q}{p}\right)^{2 k}\right) I\left(X>2^{k} t\right)$,

which is uniformly non-negative if and only if $p \leq 2$.

Proof.

By letting $\theta=0.5$, the estimator in Theorem 3.1 is written as:

$$
\begin{aligned}
\hat{V}\left(h_{1}(x ; t)\right)= & \frac{1}{p^{2}} \sum_{k=0}^{\infty}\left(2 p\left(-\frac{q}{p}\right)^{k}+(1-2 p)\left(\frac{q}{p}\right)^{2 k}\right) I\left(X>2^{k} t\right)-\sum_{k=0}^{\infty} \frac{1}{p}\left(-\frac{q}{p}\right)^{k} I\left(X>2^{k+1} t\right) \\
& \frac{1}{p^{2}}\left\{I(X>t)+\sum_{k=1}^{\infty}\left(2 p\left(-\frac{q}{p}\right)^{k}+(1-2 p)\left(\frac{q}{p}\right)^{2 k}\right) I\left(X>2^{k} t\right)\right\}- \\
& \sum_{k=0}^{\infty} \frac{1}{p}\left(-\frac{q}{p}\right)^{k} I\left(X>2^{k+1} t\right) \\
= & \frac{1}{p^{2}} I(X>t)+\sum_{k=1}^{\infty}\left\{\frac{1}{p^{2}}\left(2 p\left(-\frac{q}{p}\right)^{k}+(1-2 p)\left(\frac{q}{p}\right)^{2 k}\right)-\frac{1}{p}\left(-\frac{q}{p}\right)^{k-1}\right\} I\left(X>2^{k} t\right) \\
= & \frac{1}{p^{2}} I(X>t)+\frac{1}{p^{2}} \sum_{k=1}^{\infty}\left(-(p+2 q)\left(-\frac{q}{p}\right)^{k-1}+(1-2 p)\left(\frac{q}{p}\right)^{2 k}\right) I\left(X>2^{k} t\right) \\
= & \frac{1}{p^{2}} I(X>t)+\frac{1}{p^{2}} \sum_{k=1}^{\infty}\left(-(1+q)\left(-\frac{q}{p}\right)^{k-1}+(1-2 p)\left(\frac{q}{p}\right)^{2 k}\right) I\left(X>2^{k} t\right) .
\end{aligned}
$$


When $2^{k+1} t>X>2^{k} t$ for some $k=0,1,2, \ldots, \infty$, we simplify it as:

$$
\begin{aligned}
\hat{V}\left(h_{1}(x ; t)\right) & =\frac{1}{p^{2}}\left\{1-(1+q) \frac{1-\left(-\frac{q}{p}\right)^{k}}{1+\frac{q}{p}}+(1-2 p)\left(\frac{q}{p}\right)^{2} \frac{1-\left(-\frac{q}{p}\right)^{2 k}}{1-\left(\frac{q}{p}\right)^{2}}\right\} \\
& =\frac{1}{p^{2}}\left\{1-p(1+q)\left(1-\left(-\frac{q}{p}\right)^{k}\right)+(1-2 p) q^{2} \frac{1-\left(-\frac{q}{p}\right)^{2 k}}{p^{2}-q^{2}}\right\} .
\end{aligned}
$$

It is easy to show the following equivalents using $p+q=1$ :

$$
\begin{aligned}
& p(1+q)=(1-q)(1+q)=1-q^{2} \\
& p^{2}-q^{2}=(p+q)(p-q)=p-q=2 p-1 .
\end{aligned}
$$

Then equation 3.7 can be rewritten as:

$$
\begin{aligned}
\hat{V}\left(h_{1}(x ; t)\right) & =\frac{1}{p^{2}}\left\{1-\left(1-q^{2}\right)\left(1-\left(-\frac{q}{p}\right)^{k}\right)-q^{2}\left(1-\left(-\frac{q}{p}\right)^{2 k}\right)\right\} \\
& =\frac{1}{p^{2}}\left\{\left(1-q^{2}\right)\left(-\frac{q}{p}\right)^{k}+q^{2}\left(-\frac{q}{p}\right)^{2 k}\right\} \\
& =\frac{1}{p^{2}}\left(-\frac{q}{p}\right)^{k}\left\{\left(1-q^{2}\right)+q^{2}\left(-\frac{q}{p}\right)^{k}\right\} .
\end{aligned}
$$

It stands out that $\hat{V}\left(h_{1}(x ; t)\right) \geq 0$, if $1<p \leq 2$. This is as a consequence of $1-q^{2}>0$ and $0<-\frac{q}{p}<1$. To validate that $1<p \leq 2$ is also a necessary condition, we analysis Equation $3.8 \geq 0$ for $k \rightarrow \infty$ :

$$
\hat{V}\left(h_{1}(x ; t)\right) \stackrel{k \rightarrow \infty}{\longrightarrow} \frac{1}{p^{2}}\left(-\frac{q}{p}\right)^{k}\left(1-q^{2}\right) \geq 0 .
$$

A necessary condition for the above is $\left(1-q^{2}\right) \geq 0$, which is equivalent to $-1 \leq q<0$.

Similarly by switching the role of $p$ and $q$ and taking over $\theta$ with $\frac{1}{\theta}$, the following corollary is established. 
Corollary 3.4. When $\theta=2$ and $p<0$, an unbiased estimator of the variance of $h_{2}(x ; t)$ is

$\hat{V}\left(h_{2}(x ; t)\right)=\frac{1}{q^{2}} I(X>2 t)+\frac{1}{q^{2}} \sum_{k=1}^{\infty}\left(-(1+p)\left(-\frac{p}{q}\right)^{k-1}+(1-2 q)\left(\frac{p}{q}\right)^{2 k}\right) I\left(X>2^{k+1} t\right)$,

which is uniformly non-negative if and only if $1<q \leq 2$.

\section{Conclusion}

In this paper, we have introduced two unbiased estimators $h_{1}(x ; t)$ and $h_{2}(x ; t)$ of the reliability function $R(t)=e^{-t / \lambda}$ based on a single observation $x$ from a mixture of two exponential distributions. Further we have demonstrated that there is no proper estimator of $R(t)$ as neither being $h_{1}(x ; t)$ or $h_{2}(x ; t)$ alone, nor linear combination of $h_{1}(x ; t)$ and $h_{2}(x ; t)$, when only positive weight of the mixture distributions is permitted. This result is due to the nature of mixing properties of the two exponential distributions.

We have verified that a proper unbiased estimator can be obtained while accepting negative weights on one of the two component distributions. We have also investigated the expressions of unbiased estimators of the variances, and verified that under certain circumstance, unbiased variance estimators may turn out to be non-negative.

Based on our results, it would be interesting to further explore the existence and nature of unbiased estimator of $R(t)$ based on an observation from a general mixture of exponential distributions. Also use of one single order statistic [in a random sample from such a population] for reliability estimation would be worth pursuing.

\section{Acknowledgment}

This research is supported by the U.S. National Science Foundation under Grant DMS-1306394.

\section{References}

Basu, A., 1964. Estimates of reliability for some distributions useful in life testing. Technometrics 6 (2), 215-219. 
Jevremovic, V., 1991. A note on mixed exponential distribution with negative weights. Statistics \& probability letters 11 (3), 259-265.

Pugh, E. L., 1963. The best estimate of reliability in the exponential case. Operations research 11 (1), 57-61.

Ross, S. M., 2010. Introduction to Probability Models. Academic Press.

Sinha, B. K., Sengupta, S., Mukhuti, S., 2006. Unbiased estimation of the distribution function of an exponential population using order statistics with application in ranked set sampling. Communications in StatisticsTheory and Methods 35 (9), 1655-1670. 